\title{
Construction robotics for designed granular materials: in situ construction with designed granular materials at full architectural scale using a cable-driven parallel robot
}

\author{
Karola Dierichs $^{1}$ (1) $\cdot$ Ondřej Kyjánek ${ }^{1} \cdot$ Martin Loučka $^{1} \cdot$ Achim Menges $^{1}$
}

Received: 18 June 2019 / Accepted: 10 October 2019 / Published online: 25 October 2019

(C) The Author(s) 2019

\begin{abstract}
The article presents a cable-driven parallel robot for the in situ construction with designed granular materials at full architectural scale. Granular materials are defined as high numbers of particles larger than a micrometer, between which only short-range repulsive forces are acting. Therefore, they can have the properties of both a solid and a liquid. These materials are, thus, highly pertinent as construction materials, since they are fully recyclable and reconfigurable. Going even beyond these basic properties, a designed granular material allows to tune its overall characteristics through the design of the individual particle. Granular materials can only be deployed in situ and at full scale. Suitable robotic construction systems need to be developed. Cable-driven parallel robots are defined as robotic systems, in which an "end effector" is operated by a set of cables, which are driven by computer numerically controlled motors. The cables are running through elevated pulleys. A cable-driven parallel robot, thus, allows for a "working space", which covers an entire building site. It is comparatively lightweight and, thus, transportable between different construction sites, it is rapidly deployable, since the entire set-up takes one day only, and it is adaptable, since the pulleys can be installed in various geometric configurations. The results of this investigation show that cable-driven parallel robots are suitable as construction systems for the full-scale in situ construction of spatial enclosures with designed granular materials. This opens up a new field of research into the potentials of these full-scale, lightweight, rapidly deployable and adaptable robotic construction systems.
\end{abstract}

Keywords Designed materials · Granular materials · Cable-driven parallel robot

\section{Introduction}

Designed granular materials are an entirely new class of material systems in architecture. They consist of large numbers of custom-designed particles, which are not bound to each other but are lying only in loose contact (Andreotti et al. 2013; Duran 2000; de Gennes 1998, 1999; Law and Rennie 2015; Jaeger et al. 1996a, b). Since these granular materials can only be poured in situ on a given building site, robotic systems need to be developed, which allow for a large "working space", and which are lightweight, rapidly deployable and adaptable. The paper presents the development and the final full-scale demonstrator of such a designed

Karola Dierichs

karola.dierichs@icd.uni-stuttgart.de

1 Institute for Computational Design and Construction, University of Stuttgart, Stuttgart, Germany granular material and matching robotic system. It is the first successful validation at full architectural scale of in situ construction with designed granular materials using a cabledriven parallel robot, see Fig. 1.

Four scientific sub-questions were investigated in the context of this overarching contribution: (1) Which designed granular materials are suitable to construct spatial enclosures using a cable-driven parallel robot? (2) Which "end effector" (ISO8373 2012) is suited to distribute these designed granular materials? (3) How does the cable-driven parallel robot's hardware need to be configured for full-scale construction with designed granular materials? (4) Which robot software is suited to operate the robot using both non-"sensory" and "sensory" control (ISO8373 2012) in an open interface?

These four questions are evaluated against the following criteria as defined in ISO8373 (2012) (1) The required or specified dimensions of the "working space", (2) The required or specified "rated loads", (3) The required or specified "path accuracy". 

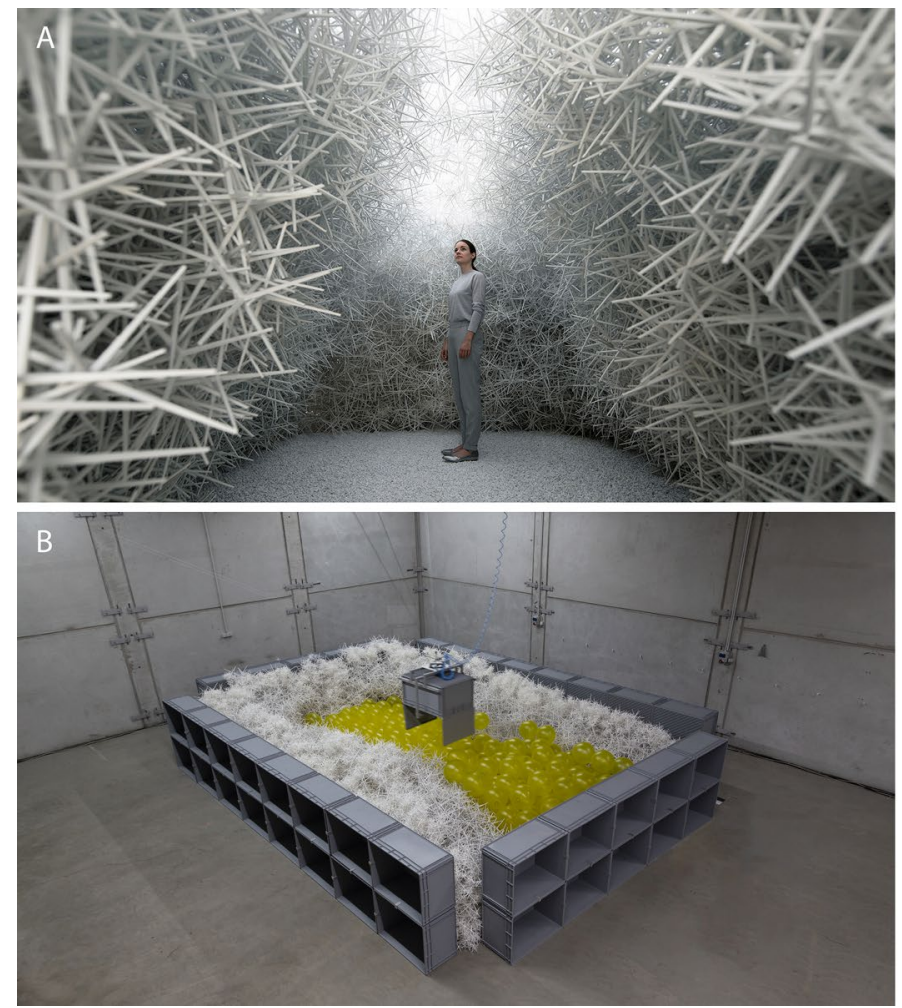

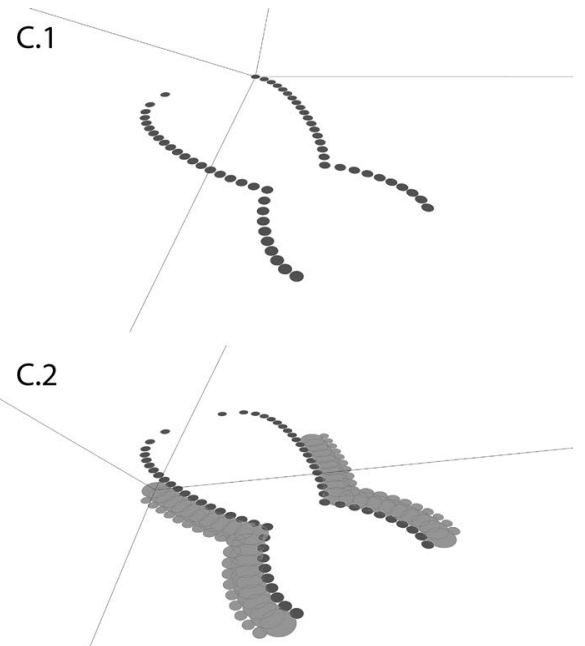

C.3

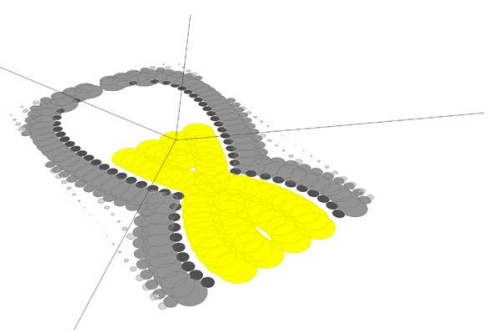

Fig. 1 ICD Aggregate Pavilion 2018. a The ICD Aggregate Pavilion 2018 is the first architectural spatial enclosure constructed with designed granular materials. b It has been constructed using a cabledriven parallel robot, which has been installed in an industrial hall. $\mathbf{c}$ The cable-driven parallel robot was controlled through a custom-written interface, which was embedded in a parametric modeling soft-

Granular materials are defined as particles in high numbers, which are in size above a micrometer. Between these particles, only short-range repulsive forces are acting (Andreotti et al. 2013; Duran 2000; de Gennes 1998, 1999; Law and Rennie 2015; Jaeger et al. 1996a, b). In nature they occur in systems such as sand or snow (Andreotti et al. 2013; de Gennes 1998, 1999; Law and Rennie 2015; Jaeger et al. 1996a, b). Granular materials are the substance which is second most handled by humans after water, and in architecture granular materials are most commonly known in their bound form as an aggregate in concrete construction (Gorse et al. 2013; Hensel et al. 2010; Hensel and Menges 2008b, a; Duran 2000; de Gennes 1998, 1999). In their unbound form, they are seldomly deployed in architectural construction (Hensel et al. 2010; Hensel and Menges 2008b, a). Yet, especially the unbound state of a granular material is highly pertinent in this specific field, as it allows to move between liquid- and solidlike states (de Gennes 1998, 1999; Jaeger et al. 1996a, b). The resulting architectural structures are, thus, entirely recyclable and reconfigurable (Hensel et al. 2010; Hensel ware. It allowed to define positions in the $\mathrm{x}-, \mathrm{y}-, \mathrm{z}$-coordinate space. Designed granular materials were deposited in layers. (C.1) The inner spatial contour was laid first with non-convex particles at high resolution. (C.2) The outer boundary was filled in with non-convex particles at low resolution. (C.3) Convex particles were filled in as a removable formwork in the core of the structure

and Menges 2008a, b; Aejmelaeus-Lindström et al. 2016; Keller and Jaeger 2016; Dierichs and Menges 2012, 2016).

If the individual particle composing a granular material is artificially made and defined in its geometry and material, one speaks of a designed granular material: the design of the particles affects the properties of the overall granular material (Dierichs and Menges 2016; Jaeger 2015; Athanassiadis et al. 2014; Hensel and Menges 2006; Matsuda 2006). Consequently, specific characteristics of the granular material can be defined, which are pertinent for the construction process or for the performance of the resulting architectural structure (Keller and Jaeger 2016; Dierichs and Menges 2015, 2016).

Designed granular materials in architecture are a relatively new area of research in architectural design and construction (Aejmelaeus-Lindström et al. 2016; Keller and Jaeger 2016; Dierichs and Menges 2016; Hensel and Menges 2006, 2008a, b). Precedent projects have been conducted at Cranbrook Academy of Art, the Architectural Association, Rice University, the Swiss Federal Institute of Technology in Zurich and the Massachusetts Institute of Technology (Aejmelaeus-Lindström et al. 2016; Dörfler et al. 2014; 
Piskorec 2014; Hensel et al. 2010; Tsubaki 2009, 2011, 2008; Hawkins and Newell 2008; Matsuda 2006, 2008; Hensel and Menges 2006, 2008a). In granular physics quite a wide range of relevant designed granular materials have been investigated (Meng et al. 2017; Murphy et al. 2016; Miskin 2016; Athanassiadis et al. 2014; Franklin 2014; Miskin and Jaeger 2013; Gravish et al. 2012; Trepanier and Franklin 2010; Malinouskaya et al. 2009; Galindo-Torres et al. 2009; Wouterse et al. 2009; Blouwolff and Fraden 2006; Philipse 1996).

The results presented in this article are part of a larger research project into "granular architectures" conducted at the Institute for Computational Design and Construction at the University of Stuttgart (Dierichs 2019).

Due to the fact that granular materials are loose, they need to be poured in situ into a desired stable state. Suitable robotic systems, thus, need to have a "working space" that covers the entire construction site, and which are lightweight, rapidly deployable and adaptable.

A set of construction systems for designed granular materials in architecture has been explored in the course of the overarching research into designed granular materials (Dierichs 2019; Dierichs and Menges 2016). These range from locally effective systems, such as six-axis robots to globally effective ones such has vibration-induced aggregation and disaggregation (Dierichs 2019; Dierichs and Menges 2016). Out of all construction systems investigated so far, cable-driven parallel robots best meet the previously mentioned performance criteria of a large "working space", a light weight, rapid installation and adaptation to various site constraints. Therefore, the aim of the research was to validate the use of these specific robots for the construction with designed granular materials through the realization of a full-scale demonstrator. Cable-driven parallel robots are defined as robotic systems, in which the flexible parallel links are driven by actuated spools, where the position of the "end effector" can be controlled by extending or retracting the cables while maintaining a positive tension in all of them (Tempel and Pott 2016; Michelin et al. 2015; ISO8373 2012; Collard et al. 2011; Merlet and Gosselin 2008; Merlet 2000; Landsberger 2005). They allow for a large "working space", which is determined by the amount and the length of the cables, the fixing height of the pulleys, the weight of the "end effector" and its moving speed (Tempel et al. 2018; Tempel and Pott 2016; Lau et al. 2016; Bruckmann and Pott 2013; Bruckmann et al. 2008; Verhoeven 2004). They are comparatively lightweight and in addition, their total packing volume is circa 2.25 cubic meters. This makes them highly transportable between different construction sites. They are also rapidly deployable with the set-up of the entire system taking circa one day. Lastly, they are adaptable to various site conditions since the motors and cable pulleys can be arranged in different configurations matching each specific construction site (Lau et al. 2016; Bruckmann et al. 2008; Albus et al. 1992), see Fig. 2. The computation of the tool center point (TCP) is mostly conducted using inverse kinematics (Tempel and Pott 2016; Merlet and Gosselin 2008). Calculations consider the cable tensions, the cable forces and the cable dynamics (Tempel and Pott 2016; Merlet and Gosselin 2008). A purely geometrical approach, such as the trilateration model deployed in this project, is rarely deployed. Yet the advantages of such a geometrical model are the ease of application and calculation speed.

The cable-driven parallel robot developed for this project has four cables and three Degrees of Freedom (DOF). An idealized mathematical model is used for the purpose of this project where the material of the cables is being considered as perfectly flexible and non-stretchable. It allows for "spatial motion of a point" as defined in Bruckmann et al. (2008). The robotic system can be classified as an Incompletely Restrained Positioning Mechanism (IRPM) (Bruckmann et al. 2008; Ming and Higuchi 1994a, b). This means that the manipulator is under-constrained, as it works only with additional wrenches acting on the "end effector" (Bruckmann et al. 2008). In the case presented here, gravity is taking the role of an additional wrench. The weight of the "end effector" is, therefore, crucial and the mechanism will not work if the mass of the "end effector" is too little. The number of cables (m) is larger than DOF (n). The additional fourth cable is not used for expanding the amount of DOF but rather to expand the "working space". By adding only one more cable, the theoretical ideal "working space", which is disregarding cable weight and slack, can be doubled.

The research presented in the following, thus, introduces new results both on the level of the designed granular material and on that of the related robotic construction system. These two aspects can only be developed and evaluated in close interrelation: the designed granular material becomes an integral part of the construction system and the construction system is developed to suit the requirements of the specific designed granular material. Eventually, the tendency would be to even further merge the particle- and the construction system, with the particles becoming more and more construction machines in themselves and the construction system becoming more and more minimally integrated into the particle system.

\section{Results}

Designed granular materials can only be poured in situ on a given building site. Thus, robotic systems need to be developed, which allow for a large "working space", which are lightweight, rapidly deployable and adaptable to different site conditions. The paper presents the development and the 


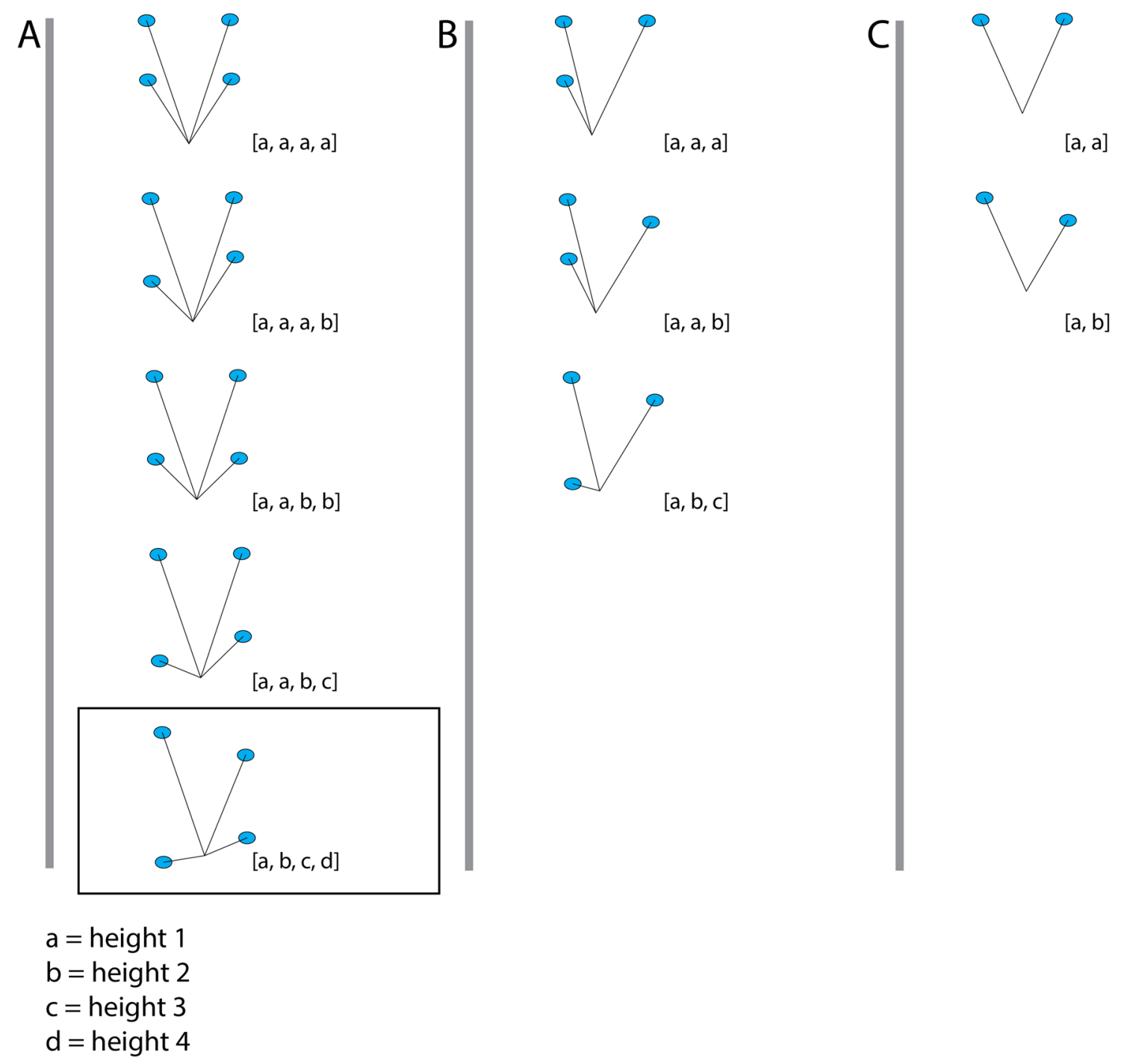

Fig. 2 Different configurations of the cable-driven parallel robot. a Combinations in different pulley heights for the four motors set-up. The highlighted configuration [a, b, c, d] is explained in greater detail

realization of a full-scale demonstrator of a designed granular material and a custom-built robotic system.

\subsection{Sub-question 1: designed granular material}

Two different types of designed granular materials were deployed for this project: one consisting of non-convex particles and one consisting of convex ones. Non-convex particles allow for increased stability in a granular material and are thus suited for the formation of domes, vaults and arches. Convex particles render a granular material less stable and consequently can be deployed as a removable formwork.

The non-convex particles are mainly hexapods. Their precise geometry has been developed through statistical series, which relate packing density of a granular material to the in Fig. 8. b Combinations in different pulley heights for the three motors set-up. c Combinations in different pulley heights for the two motors set-up

composing particles' geometry. The hexapods have a diameter of $300 \mathrm{~mm}$. They are fabricated using injection molding and are made from recycled plastics. One particle weighs circa $30 \mathrm{~g}$, see Fig. 3. The hexapods are partially mixed with decapods. The decapods have a diameter of $115 \mathrm{~mm}$ and weigh circa $5 \mathrm{~g}$ each. They have also been produced with injection molding.

The convex particles are inflatable spheres with a diameter of circa $260 \mathrm{~mm}$, see Fig. 3. They are inflatables, which are made from polyvinyl chloride (PVC) foil. Other than in this case study, they do not need to be sourced from industry but also could be custom made to further calibrate the characteristics of the granular material, which is consisting of them. To form a visual contrast for image segregation, the convex particles are 

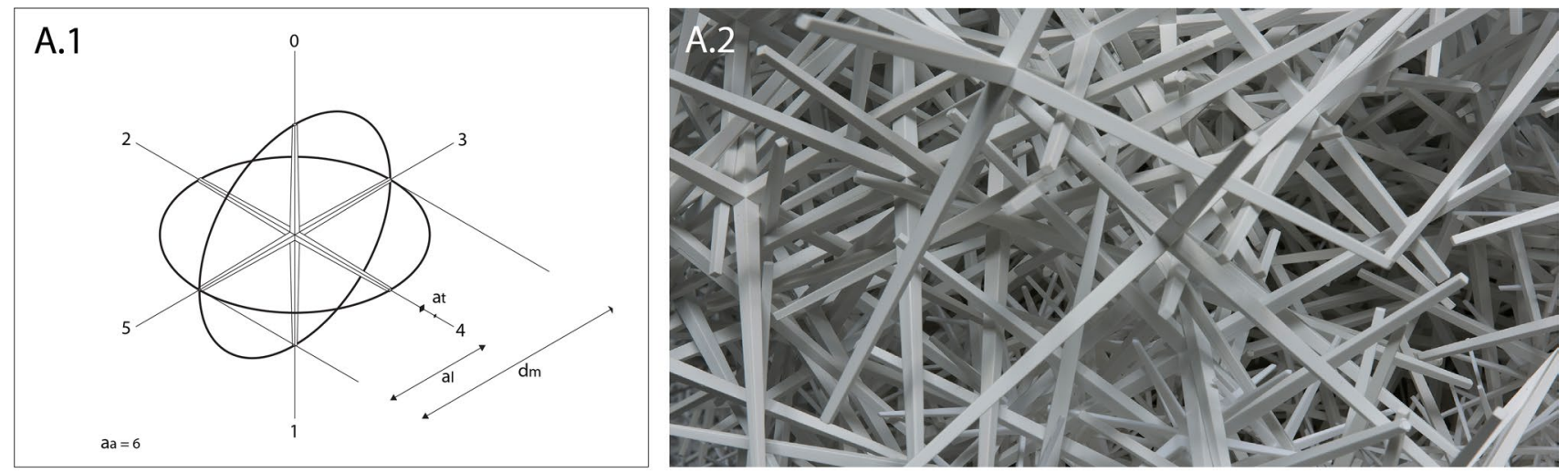

B.1
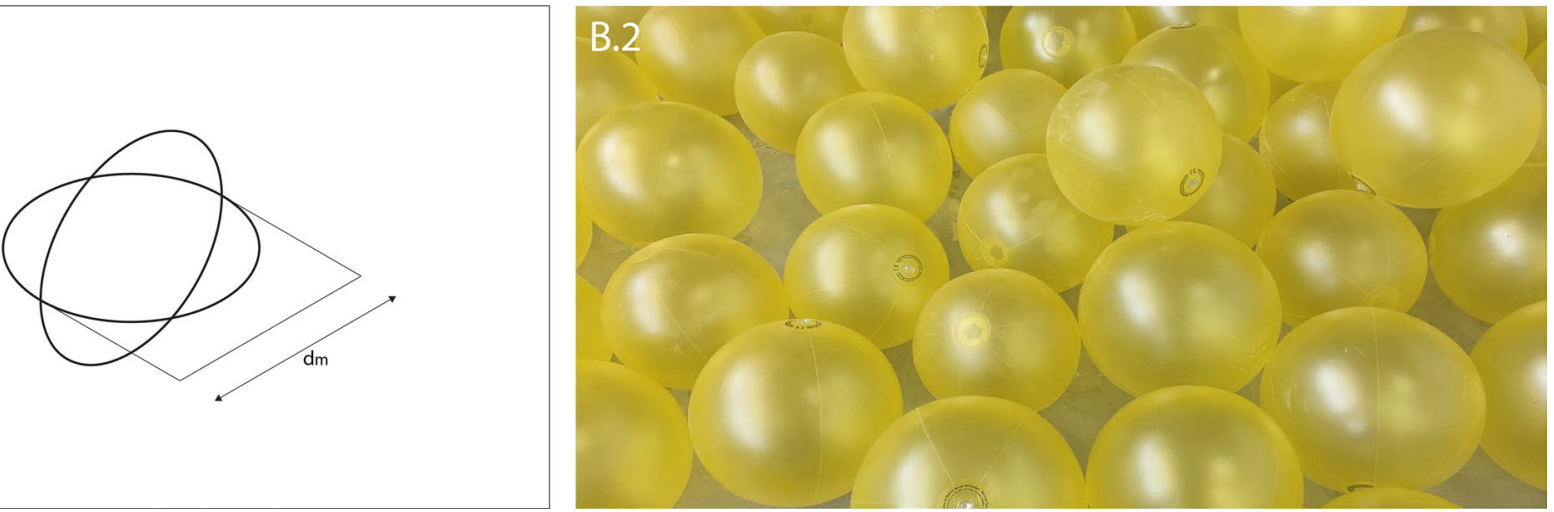

Fig. 3 Non-convex and convex particles. a Hexapods (A.1) The hexapods are based on a parametric model, which allows to specify longest diameter of the convex hull (dm), the arm amount (aa), the axis length (al) as well as the arm taper (at). (A.2) The hexapods interlock and can thus form stable structures. b Spheres (B.1) The spheres are defined by their diameter $(\mathrm{dm})$ only. Here diameter of the convex hull and particle geometry coincide. (B.2) The spheres do not interlock and can be used as a formwork, which can flow out of the structure chosen in yellow and the non-convex particles are white. The particles have been tested for packing density, arch formation and coordination number.

The two particle geometries can be evaluated against the three criteria of "working space", "path accuracy" and "rated load". The required "working space" is affected by the total volume of the available granular material, which is circa $155-120$ cubic meters for about 70,000 hexapods and circa 12 cubic meters for roughly 50,000 decapods. The spheres, which form a negative volume, are not considered in this assessment of the "working space" in order to allow for a safety margin. In principle, the entire built volume could, thus, be filled with non-convex particles. The minimum path width, that the material allows for is in the range of $150 \mathrm{~mm}$ respectively $130 \mathrm{~mm}$, which is the radius of the convex hull of the hexapods and the spheres. In combination with the "path accuracy" of the robot, this affects the overall accuracy of the construction process. The maximum load added by the particle to the construction system's "rated load" is around 3-4 kg for a full large box of either hexa- or decapods.

\subsection{Sub-question 2: "end effector"}

The storage boxes of the designed granular material are becoming part of the "end effector", which dispenses the particles, see Fig. 4. For that purpose, a frame is fitted to the flange of the cable-driven parallel robot, which allows to hold two different box sizes: 800 by 600 by $420 \mathrm{~mm}$ and 600 by 400 by $320 \mathrm{~mm}$. The boxes are fitted to the frame with their opening lid to the bottom. The lid is held by two clamps, which can be opened and closed by a pneumatically actuated parallel gripper. The entire "end effector", thus, consists of the pneumatically actuated gripper, the aluminum frame and the storage boxes.

The "end effector" without boxes weighs circa $10 \mathrm{~kg}$, the large box weighs circa $10 \mathrm{~kg}$ and the small box circa $4 \mathrm{~kg}$.

Four different plot styles were established: a large box with mixed decapods and hexapods, a large box filled with hexapods only, a small box with hexapods and a large box with spheres. Several tests were conducted to define the density of the drop pattern for the different box sizes and fillings. The two "end effector" sizes can be evaluated 


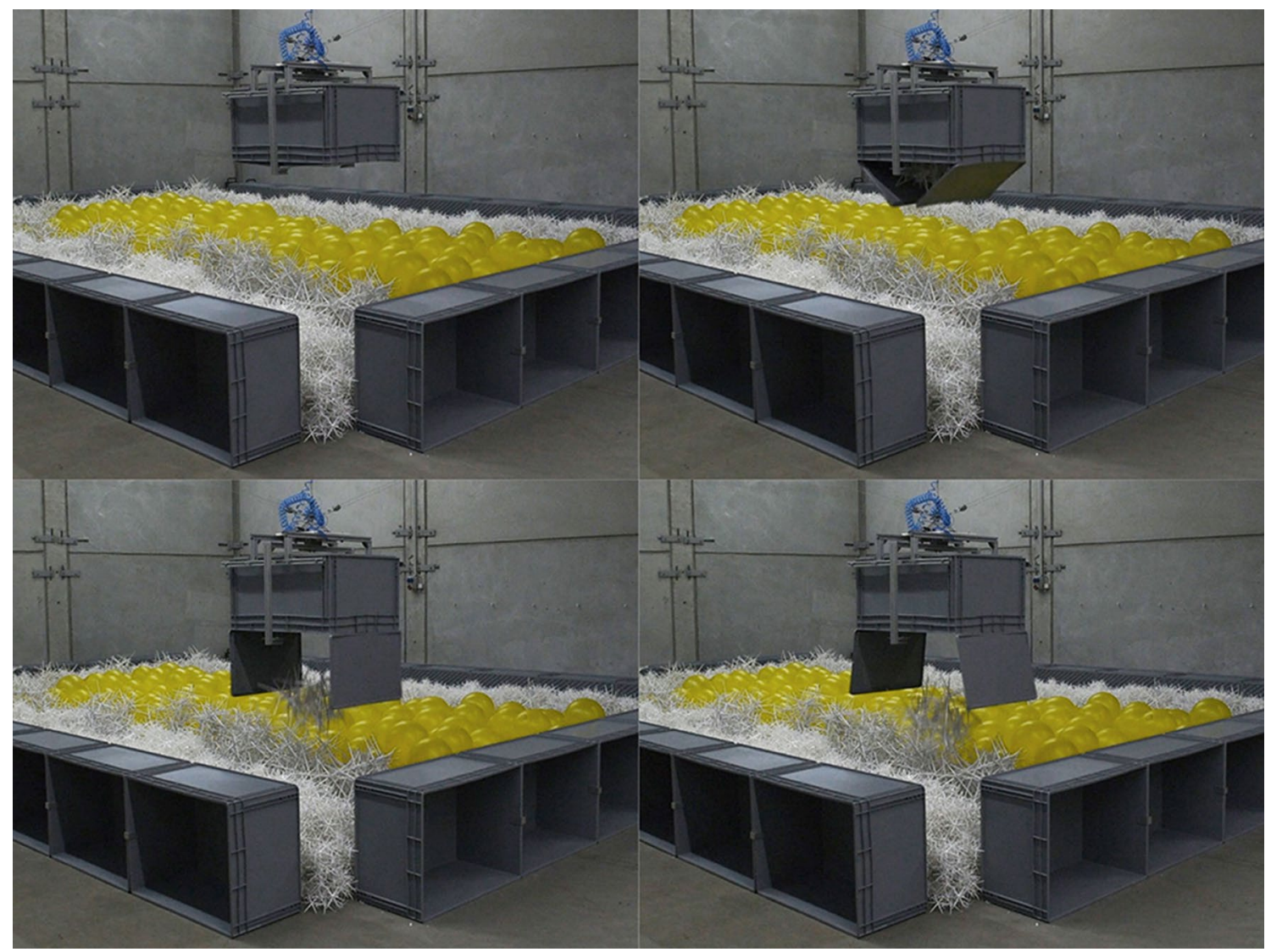

Fig. 4 "End effector". The particles are dropped directly from their storage boxes, which are fitted to the flange of the cable-driven parallel robot. The release of the lid is controlled by a parallel pneumatic gripper

against the three criteria of "working space", "path accuracy" and "rated load". The "working space" of the robot is sub-divided into the modular sizes of the "end effector" boxes. The accuracy, with which the boxes are depositing the particles, is given by their width vertical to the robot's direction of travel. This plot width is $600 \mathrm{~mm}$ in both cases. Sway of the "end effector" is accommodated for by overlapping neighboring plot lines. The "rated loads" of the robot were developed to match the requirements of the filled large box "end effector". This was mainly achieved by doubling the potential "rated load" per cable, see Sect. 2.3.

\subsection{Sub-question 3: robot hardware}

The cable-driven parallel robot is a highly dynamic system and the "path accuracy", "working space" and "rated load" depend strongly on the actual setup and the speed, at which the system is operated. The system is, therefore, demonstrated referring to the setup deployed for this project.

The cable-driven parallel robot consists of 5 sub-elements, see Fig. 5. These are the control cabinet, the four winches, the pulleys, the steel cables and the flange. The key elements of the control cabinet are the CNC controller, the frequency inverters, solid-state relays and the stepper motor drives. The four winches are driven by one 750 Watts (W) alternating current (AC) servo motor each. They have a reel drum, a reel drum bearing and a pressure cylinder. Each winch is equipped with an Electromechanical Brake (EMB4). The winches are encased in custom-made base constructions, which allow to anchor them to the ground with weights. The pulleys consist of a pulley wheel with a custom-made rotary wheel holder and a bearing. These are fixed into a custom-made pulley base, which allows to mount the pulleys to structures on site. The steel cables have a length of $51 \mathrm{~m}$ and a thickness of $1.8 \mathrm{~mm}$. Two "end effector" flanges were custom made for the robot: one for a threemotor setup and one for a four-motor setup.

The robot hardware can be evaluated against the criteria of "path accuracy", "working space" and "rated loads". The key challenge with respect to "path accuracy" is the stretch of the cables under the load and vibrations during the operation. On the one hand, those issues could be reduced using a cable material with little stretch as well as using thicker cables so that the material stretching does not influence the whole system. On the other hand, operating under lower speed allowed to decrease vibrations and the spring effect of 


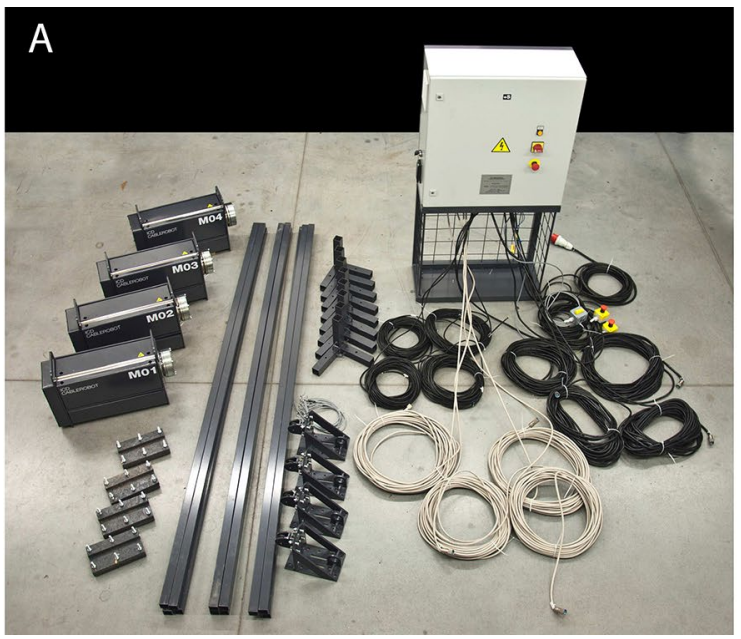

Fig. 5 Robot hardware. a The robot hardware consists of the control cabinet, the winches, the pulleys, the steel cables and the flange. b The assembled robot hardware requires the operator to be seated

the cables. In future applications, the "path accuracy" could be further increased by attaching a stabilizing mechanism to the "end effector", such as a gyroscope.

The dimensions of the "working space" were increased horizontally, that means in $\mathrm{x}$ - and $\mathrm{y}$ - direction, by extending the distance between the pulleys and vertically in z-direction by using higher fixing points for the pulleys. As a further development with respect to an increased "working space", several cable-driven parallel robots could be grouped to work in clusters.

The potential "rated load" has been increased by switching from a fixed pulley system to a block and tackle and thus theoretically doubling the available "rated load" per cable. The "rated load" could be further enhanced by increasing the performance of the setup through the use of stronger motors, cables, gearboxes and so forth. Additional hardware mechanisms like additional pulleys and winches or the block and tackle system deployed in this case study can help to increase "rated load".

\subsection{Sub-question 4: robot software}

The robot software consists of four servo drives controlled by Pulse Width Modulation (PWM) signal connected to a computer numerically controlled (CNC) card that can be programmed with an external system by sending move data via Universal Serial Bus (USB) or Ethernet connection.

There are two different approaches for trajectory planning of the cable-driven parallel robot: The trajectory can be either planned beforehand in offline mode, stored in a plain text using a G-code syntax and later translated by a CNC G-code translator (CNCUSB software) into machine instructions; or it can be planned in real-time using a

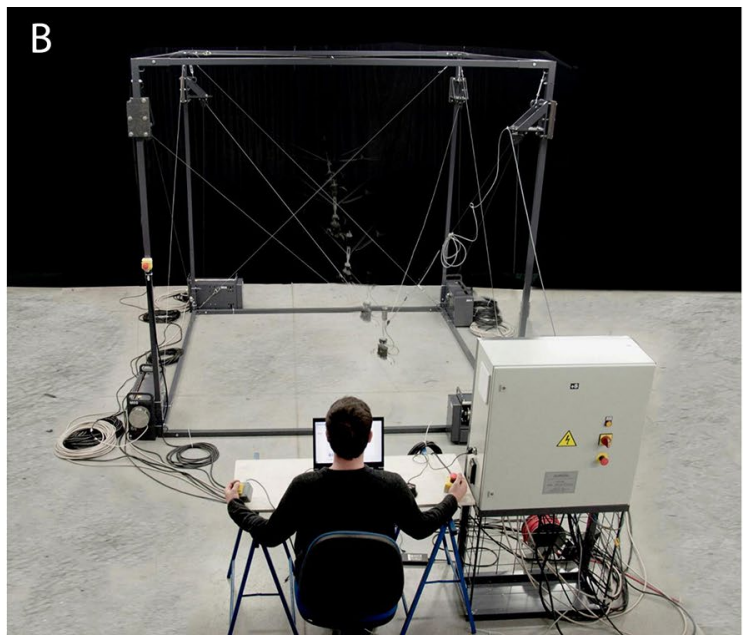

close to the control cabinet. The pulleys are mounted in an elevated position on top of the winches. The flange is mounted in the intersection of all four steel cables inside the "working space"

Custom Server Controller (CSC) that is able to communicate with external clients via User Datagram Protocol (UDP) or Websocket protocol. The server is parsing the movement commands from the clients and sending them to the movement buffer of the CNC controller card via an Ethernet connection. The latter method allows a better integration of various sensors as well as modifying the robot path during execution.

The project presented in this article has been conducted using the custom server controller (CSC). The CSC maintains cyclic communication with the $\mathrm{CNC}$ controller card reading the real-time information about the robot and sending the corresponding movement and input/output (IO) commands to the command buffer of the $\mathrm{CNC}$ controller card.

The communication with the external clients is based on a publisher-subscriber pattern using either UDP or Websocket protocol. The CSC, therefore, functions as a mediator layer between the CNC controller card and clients that are interested in controlling the robot or simply want to listen to the real-time status messages. As soon as a client subscribes, it starts to receive a real-time status messages and can send control commands. Clients that did not send a control command or a sign-of-life message for a specific period of time, which has been set to 2000 milliseconds (ms) in this case, will get automatically unsubscribed from the list.

Communication between the subscribers and the server is provided by sending simple JavaScript Object Notation (JSON) encoded messages. Communication between the server and the CNC controller card is a subject to a proprietary library shipped with the specific controller card used. The library has an exposed public Advanced Programming Interface (API) but it is not open sourced. The server controller can send a linear motion to the robot either in an 
absolute coordinate system of the robot or in relative delta movements with respect to the current position.

As the CNC card can processes only movements in the axis coordinate space each linear movement has to be divided to an axis-specific movement with some resolution. The movement is, therefore, only linear with certain precision.

The inverse kinematics of the robot is calculated based on the angular position of the servo motors provided by the resolvers. From these data, a length of the cable can be obtained and later translated to a distance between the "end effector" and the anchor of each cable that has a known position, see Fig. 6.

To simplify the calculation, the system is being considered as an ideal system in a static or quasi-static state with all cables being stretched, that means without slack. A geometric trilateration method is being used for calculating the Cartesian position of the "end effector". This is implementing an established calculation based on the Pythagorean theorem, which is using the intersection of three spheres with known radii and centers. Performing the calculation based on four anchors instead of just three has the advantage of determining the error during the initial system calibration, that means the position of the anchors and initial length of the cables.

The basic algorithm is based on a sphere-sphere intersection as outlined in Weisstein (2019a, b).

$x^{2}+y^{2}+z^{2}=R^{2}$

$(x-d)^{2}+y^{2}+z^{2}=r^{2}$

The combination of Eqs. 1 and 2 results in:

$(x-d)^{2}+\left(R^{2}-x^{2}\right)=r^{2}$

Equation 3 can be rewritten as:

$x^{2}-2 d x+d^{2}-x^{2}=r^{2}-R^{2}$

Equation 4 can be solved for $x$ :

$x=\frac{d^{2}-r^{2}+R^{2}}{2 d}$

Such an idealized model, however, does not fully correspond with the real-life setup where a cable slack, cable flexibility and the weight of the "end effector" play a significant role for a IRPM type of cable-driven robot, such as the one used in this project. A valid solution that can improve precision
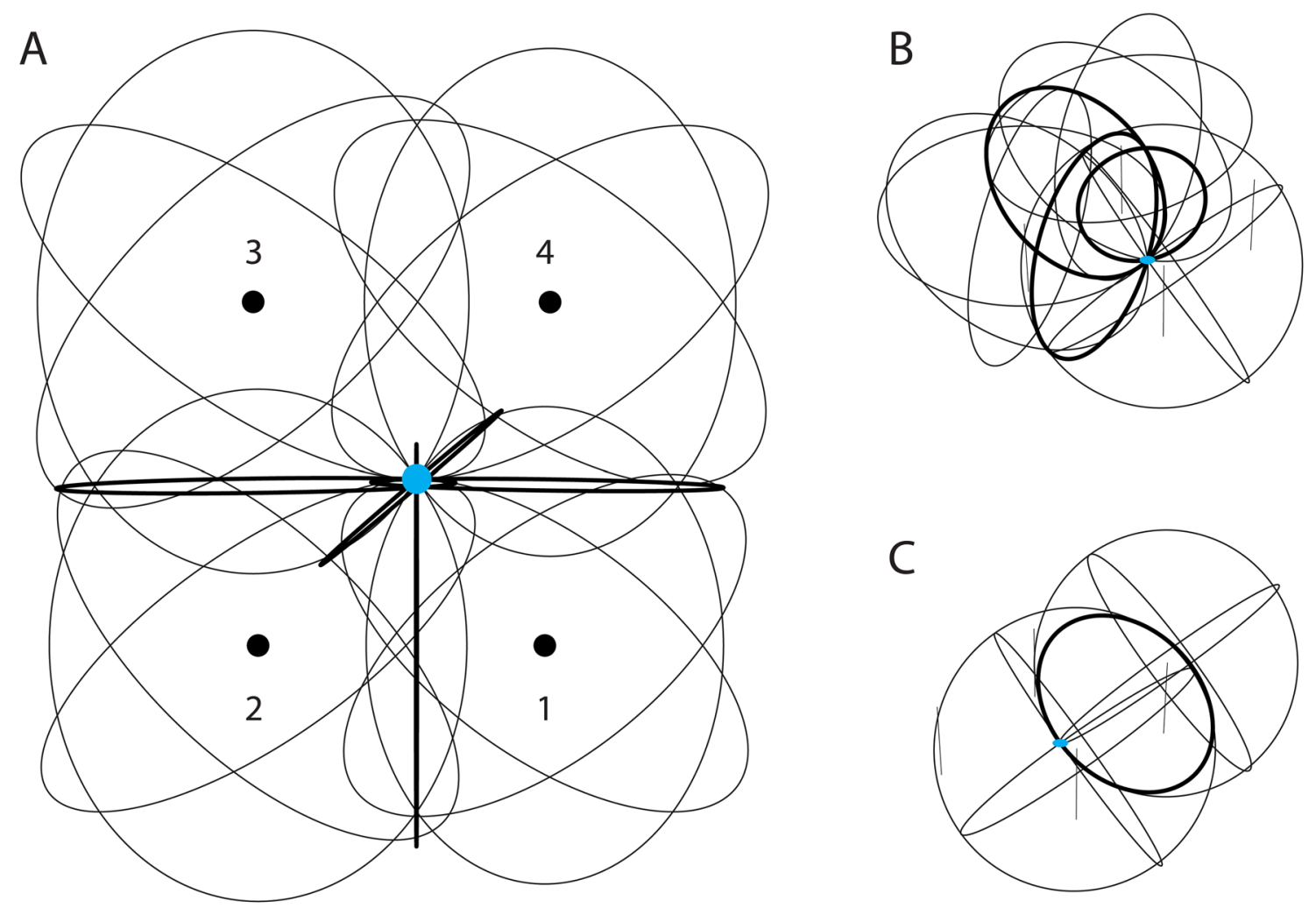

Fig. 6 Trilateration. a The trilateration algorithm computes the cable lengths based on the desired location of the tool center point (TCP) through the intersection of four spheres, which have their midpoints in the four anchors of the pulleys. b The intersection of three spheres renders the lengths of the cables based on the position of the TCP. c The calculation can be double-checked by the intersection of the fourth sphere with the result of the trilateration 
significantly especially for larger spans is to minimize cable stretch and include a slack rope model in the TCP position calculation li et al. (2013). Such optimization cannot only improve positional accuracy but also can help to define the "working space" of the cable-driven robot setup more precisely. Another useful addition to the current software solution would be the calculation of a load distribution on cables based on the position of the robot TCP within a "working space" of the robot. As a load distribution changes with changing cable length and angle, the effective "rated load" of the overall setup changes as well. By calculating load distribution, it is, therefore, possible to further improve the determination of a possible "working space" with respect to an actual weight of the "end effector" and attached workpiece.

However, with respect to the evaluation criteria of "path accuracy", "working space" and "rated load", the proposed model offers an approach, which is sufficiently accurate for the purpose of construction with designed granular materials, while at the same time being fast to compute and relatively simple to implement. This is due to the fact that these construction materials do not require very high degrees of precision.

A

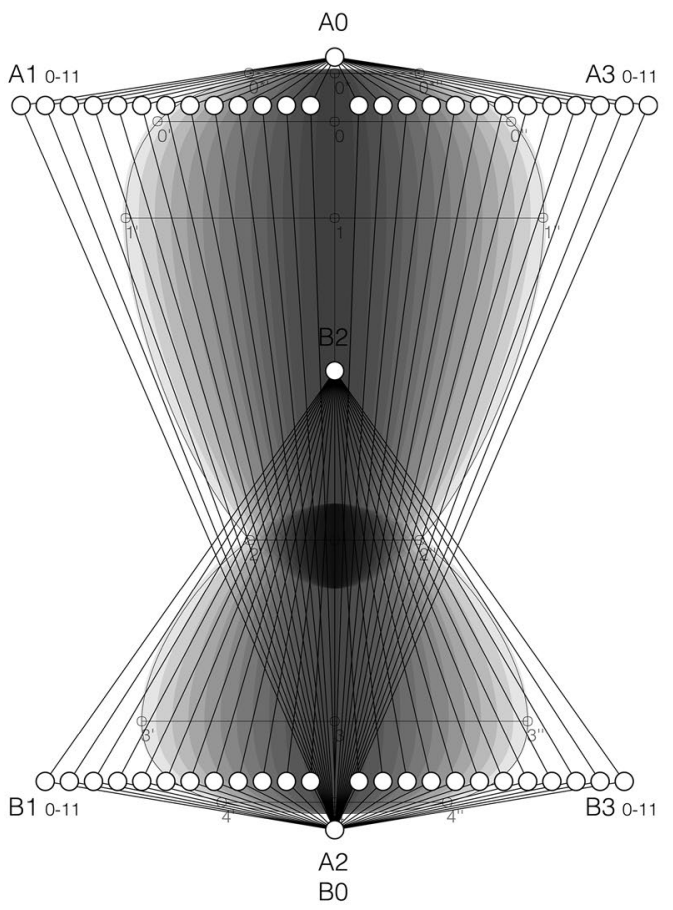

\subsection{Integrated project}

The realized project, the ICD Aggregate Pavilion 2018, integrated the sub-aspects of the system: the designed granular material, the "end effector", the robot hardware and its software.

The pavilion was constructed in horizontal layers. A fixed pickup point was given for loading and unloading of the box "end effector". Altogether four different plot styles were deployed: three for non-convex particles with different box sizes and particle mixes and one for convex ones. The nonconvex particles were laid first, and the convex ones were deposited in the core of the structure, see Fig. 7.

The drop pattern was adjusted to deviations in height during construction. The entire process was surveyed using image segregation. For that purpose, the software allowed the integration of image data obtained from a remote camera, which was fixed on top of the construction space. The image data were used as a base for the image segregation. This process allowed to filter out yellow values of the convex spheres and consequently adjust the geometry of the structure, in case there were outlying particles.
B

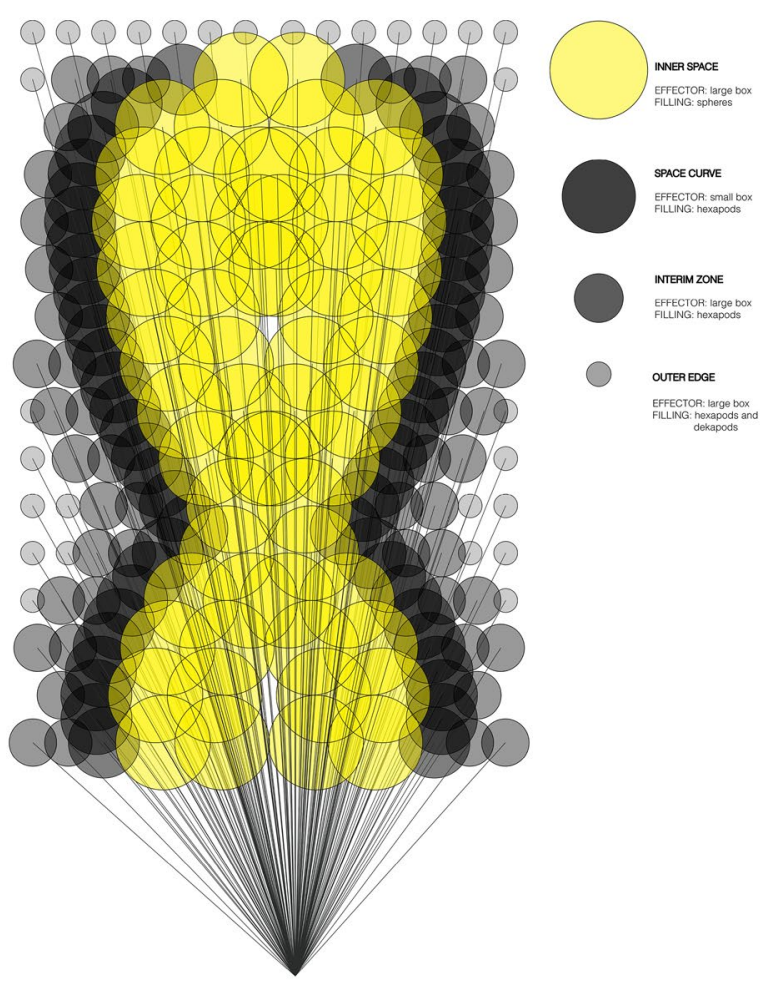

Fig. 7 Design model and drop pattern. a The design model consists of two intersecting closed Non-uniform Rational B-Splines (NURBS). These are layered in height with varying width and constant length. b The drop pattern is based on the design model and allows for four different plot styles: Large boxes with spheres, small boxes with hexapods, large boxes with hexapods and large boxes with hexapods and decapods 
One of the greatest challenges was the "rated load" of the cable-driven parallel robot, which decreased with increasing height of the structure and which was lower at the edges, see Fig. 8. This required to switch to the use of small "end effector" boxes only in the upper layers.

During construction, the boxes were used as a container on the outside of the pavilion. Once construction was completed, these containing boxes were removed and the convex particles flowed out, respectively, could relatively easily be removed, if they were clogged inside the structure. Altogether, circa 1,20,000 non-convex particles and 725 convex ones were deployed. The structure weighed around $2300 \mathrm{~kg}$.

\section{Discussion}

Cable-driven parallel robots can be deployed to construct with designed granular materials for full-scale architectural structures, the calibration of "path accuracy", "working space" and "rated loads" needs to be conducted integrating all system components: the designed granular material, the "end effector", the robot hardware itself, and its software. This implies that even larger-scale structures can be constructed using cable-driven parallel robots either with a larger "working space" or combining several robots. Due to being lightweight and rapidly deployable, these constructions systems can be easily transported from one construction site to the next. Their configuration is adaptable to different site configurations, using four, three or even just two motors. Cable-driven parallel robots are thus highly suited for in situ construction in general and with designed granular materials in specific. In the following, the results are discussed with respect to the three evaluation criteria of "path accuracy", "working space" and "rated load" in the system categories of the designed granular material, the "end effector", the robot hardware and its software.

The "path accuracy" can be tuned towards both less control with respect to the designed granular material and towards more or more refined control on the robot hardware and software. This implies that the designed granular materials also allow for emergent behavior, with little or no control of the geometric outcome. This would need to be coupled with a very precise cable-driven parallel robot using additional motors and cables as well as a design model that is based on the emergent behavior of the designed granular material, potentially integrating machine learning.

The "working space" for designed granular materials is indefinite as the granular materials can be expanded by simply adding more and more particles. The robot hardware can meet this demand by increasing cable length, by combining several cable-driven parallel robots or by moving one robot along the construction site.

The "rated load" needs to be increased so that the box "end effectors" can become larger for areas of the structure which does not need a high resolution. This will rapidly decrease construction time. Increasing the "rated load" of
A

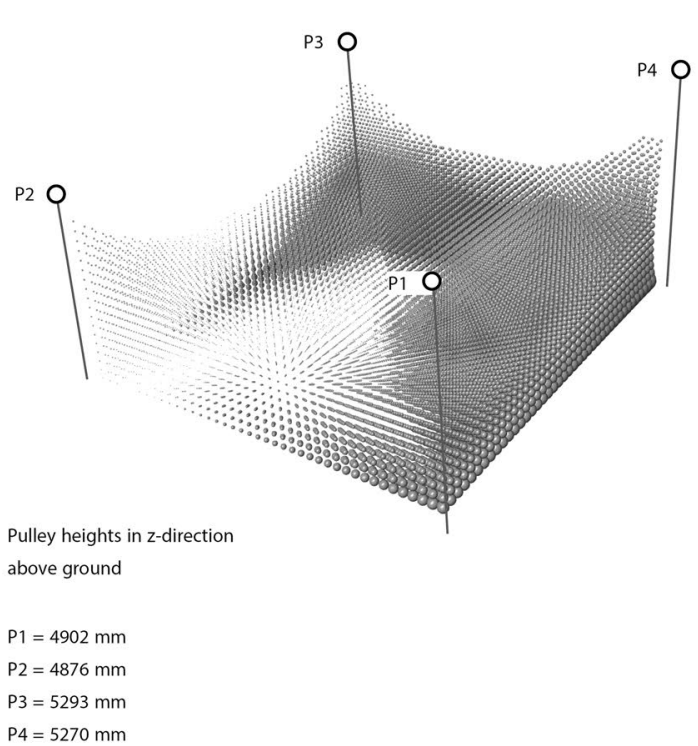

B

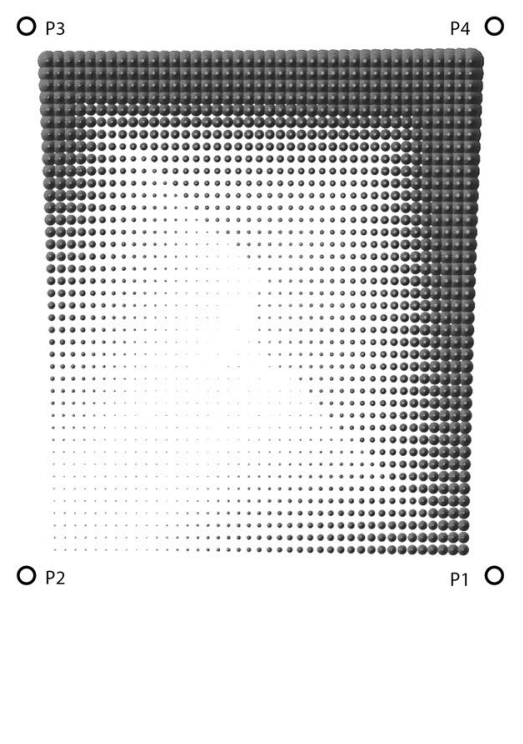

C

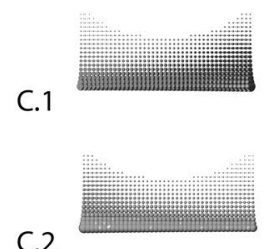

C.2

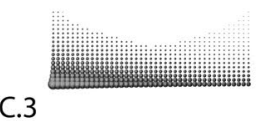

C.4 space", which can be reached with the load of a large box "end effector". b Top view of the points in reach with a large box "end effector". c Side views of the same points. (C.1) Front view, (C.2) Back view, (C.3) Right view, (C.4) Left view 
the robot implies larger motor, higher fixing points of the pulleys or the multiplication of pulley in one cable. The designed granular material can be developed for low packing and high deployed volume so that the "rated load" of the construction system is used to achieve as much built volume as possible.

Further research will, thus, be directed towards merging the designed granular material and its construction system towards an even higher level of integration.

\section{Acknowledgements The research has been funded by the GETTYLAB} and ITASCA INTERNATIONAL Inc.

Author contributions KD has led the research on designed granular materials, has conceived of the demonstrator project and conducted the construction process. OK has developed the software interface of the cable-driven parallel robot and contributed to its hardware development. ML has conceived of and constructed the cable-driven parallel robot and contributed to its software development. AM has supervised the research on designed granular materials and related construction systems. Research assistants of the ICD Aggregate Pavilion 2018 were: Christian Arias, Bahar Al Bahar, Elaine Bonavia, Federico Forestiero, Pedro Giachini, Shir Katz, Alexandre Mballa-Ekobena, Leyla Yunis and Jacob Zindroski.

\section{Compliance with ethical standards}

Conflict of interest The authors declare that they have no conflict of interest.

Open Access This article is distributed under the terms of the Creative Commons Attribution 4.0 International License (http://creativeco mmons.org/licenses/by/4.0/), which permits unrestricted use, distribution, and reproduction in any medium, provided you give appropriate credit to the original author(s) and the source, provide a link to the Creative Commons license, and indicate if changes were made.

\section{References}

Aejmelaeus-Lindström P, Willmann J, Tibbits S, Gramazio F, Kohler M (2016) Jammed architectural structures: towards large-scale reversible construction. Granul Matter 18(2):28. https://doi. org/10.1007/s10035-016-0628-y

Albus JS, Bostelman RV, Dagalakis NG (1992) The nist robocrane. J Robot Syst 10(5):373-385

Andreotti B, Forterre Y, Pouliquen O (eds) (2013) Granular media: between fluid and solid. Cambridge University Press, Cambridge

Athanassiadis AG, Miskin MZ, Kaplan P, Rodenberg N, Lee SH, Merritt J, Brown E, Amend J, Lipson H, Jaeger HM (2014) Particle shape effects on the stress response of granular packings. Soft Matter 10(1):48-59. https://doi.org/10.1039/C3SM52047A

Blouwolff J, Fraden S (2006) The coordination number of granular cylinders. Europhys Lett (EPL) 76(6):1095-1101. https://doi. org/10.1209/epl/i2006-10376-1

Bruckmann T, Pott A (2013) Cable-driven parallel robots, mechanisms and machine science, vol 12. Springer Berlin Heidelberg, Berlin, Heidelberg. https://doi.org/10.1007/978-3-642-31988-4

Bruckmann T, Mikelsons L, Brandt T, Hiller M, Schramm D (2008) Wire Robots Part I: kinematics, analysis \& design. In: Ryu J-H (ed) Design and Prototyping of a spherical parallel machine based on 3-CPU kinematics. INTECH Open Access Publisher, pp 109132. https://doi.org/10.5772/5365

Collard JF, Lamaury J, Gouttefarde M (2011) Dynamics modelling of large suspended parallel cable-driven robots multibody model. Proc ECCOMAS Themat Conf Multibody Dyn 2011:3-5

de Gennes PG (1998) Reflections on the mechanics of granular matter. Phys A: 261(3):267-293. https://doi.org/10.1016/S0378 $-4371(98) 00438-5$

de Gennes PG (1999) Granular matter: a tentative view. Rev. Mod. Phys. 71(2):S374-S382. https://doi.org/10.1103/RevModPhys .71.S374

Dierichs K (2019) Granular architectures: granular materials as 'designer matter' in architecture. PhD, University of Stuttgart, Stuttgart. (accepted thesis, publication in process)

Dierichs K, Menges A (2012) Aggregate structures: material and machine computation of designed granular substances. Architect Des 82(2):74-81. https://doi.org/10.1002/ad.1382

Dierichs K, Menges A (2015) Granular morphologies: programming material behaviour with designed aggregates. Architect Des 85(5):86-91. https://doi.org/10.1002/ad.1959

Dierichs K, Menges A (2016) Towards an aggregate architecture: designed granular systems as programmable matter in architecture. Granul Matter 18(2):25. https://doi.org/10.1007/s 1003 5-016-0631-3

Dörfler K, Ernst S, Piskorec L, Willmann J, Helm V, Gramazio F, Kohler M (2014) Remote material deposition: Exploration of reciprocal digital and material computational capacities. In: M. Voyatzaki (ed.) What's the matter materiality and materialism at the age of computation, pp. 361-377. Barcelona, Spain

Duran J (2000) Sands, Powders, and Grains: An Introduction to the Physics of Granular Materials, 1 edn. Partially Ordered Systems. Springer-Verlag, New York. https://doi. org/10.1007/978-1-4612-0499-2

Franklin SV (2014) Extensional rheology of entangled granular materials. Europhys Lett 106(5):58004. https://doi. org/10.1209/0295-5075/106/58004

Galindo-Torres SA, Alonso-Marroquìn F, Wang YC, Pedroso D, Muñoz Castaño JD (2009) Molecular dynamics simulation of complex particles in three dimensions and the study of friction due to nonconvexity. Phys Rev AE 79:060301. https://doi. org/10.1103/PhysRevE.79.060301

Gorse C, Johnston D, Pritchard M (2013) Aggregate. In: Gorse C, Johnston D, Pritchard M (eds) A dictionary of construction. Surveying and civil engineering. Oxford University Press, Oxford (Online version)

Gravish N, Franklin SV, Hu DL, Goldman DI (2012) Entangled granular media. Phys Rev Lett 108(20):208001. https://doi. org/10.1103/PhysRevLett.108.208001

Hawkins A, Newell C (2006) Aggregates 02: 2004 [project description]. In: Hensel M, Menges A (eds) Morpho-ecologies. AA Publications, London, pp 274-283

Hawkins A, Newell C (2008) Aggregat gefertigter partikel 02. archplus, form follows performance: zur wechselwirkung von material, struktur. Umwelt 41(188):82-85

Hensel M, Menges A (2006) Material systems-introduction: 20 proto-architectures, research and design projects. In: Hensel M, Menges A (eds) Morpho-ecologies. AA Publications, London, pp 62-67

Hensel M, Menges A (2008a) Aggregates. Architect Des 78(2):80-87. https://doi.org/10.1002/ad.645

Hensel M, Menges A (2008b) Materialsysteme 05: aggregate. Archplus, form follows performance: zur wechselwirkung von material, struktur. Umwelt 41(188):76-77

Hensel M, Menges A, Weinstock M (2010) Chapter 12: aggregates. In: Hensel M, Menges A, Weinstock M (eds) Emergent technologies 
and design: towards a biological paradigm for architecture. Routledge, Abingdon, pp 227-242

ISO8373: Robots and robotic devices—vocabulary (2012)

Jaeger HM (2015) Celebrating soft matter's 10th anniversary: toward jamming by design. Soft Matter 11(1):12-27. https://doi. org/10.1039/C4SM01923G

Jaeger HM, Nagel SR, Behringer RP (1996) Granular solids, liquids, and gases. Rev. Mod. Phys. 68(4):1259-1273. https://doi. org/10.1103/RevModPhys.68.1259

Jaeger HM, Nagel SR, Behringer RP (1996) The physics of granular materials. Phys Today 49(4):32-38. https://doi. org/10.1063/1.881494

Keller S, Jaeger HM (2016) Aleatory architectures. Granul Matter 18(2):29. https://doi.org/10.1007/s10035-016-0629-x

Landsberger SE (2005) Design and construction of a cable-controlled, parallel link manipulator. Thesis (m.s.), Massachusetts Institute of Technology

Lau D, Eden J, Tan Y, Oetomo D (2016) Caspr: a comprehensive cablerobot analysis and simulation platform for the research of cabledriven parallel robots. In: IROS 2016, pp. 3004-3011. IEEE, [Piscataway, New Jersey]. https://doi.org/10.1109/IROS.2016.77594 65

Law J, Rennie R (2015) Granular material. In: Law J, Rennie R (eds) A dictionary of physics. Oxford University Press, Oxford (Online version)

li H, Zhang X, Yao R, Sun J, Pan G, Zhu W (2013) Optimal force distribution based on slack rope model in the incompletely constrained cable-driven parallel mechanism of fast telescope. Mech Mach Sci pp 87-102. https://doi.org/10.1007/978-3-642-31988-4_6

Meng Lingyi, Li Shuixiang, Yao Xiaohu (2017) Maximally dense random packings of intersecting spherocylinders with central symmetry. Powder Technol 314:49-58. https://doi.org/10.1016/j.powte c.2016.07.059

Malinouskaya I, Mourzenko VV, Thovert JF, Adler PM (2009) Random packings of spiky particles: geometry and transport properties. Phys Rev E 80(1):011304. https://doi.org/10.1103/PhysR evE.80.011304

Matsuda E (2006) Aggregates 01: 2003-2004 [project description]. In: Hensel M, Menges A (eds) Morpho-ecologies. AA Publications, London, pp 262-271

Matsuda E (2008) Aggregat gefertigter partikel 01. archplus, form follows performance: zur wechselwirkung von material, struktur. Umwelt 41(188):80-81

Merlet JP (2000) Parallel robots. Springer, Netherlands, Dordrecht

Merlet JP, Gosselin C (2008) Parallel mechanisms and robots. In: Siciliano B, Khatib O (eds) Springer handbook of robotics. Springer Science+Business Media, Berlin, Heidelberg, pp 269-285

Michelin M, Baradat C, Nguyen DQ, Gouttefarde M (2015) Simulation and control with xde and matlab/simulink of a cable-driven parallel robot (cogiro). In: Pott A, Bruckmann T (eds) Cabledriven parallel robots. Springer International Publishing, Cham, pp 71-83

Ming A, Higuchi T (1994) Study on multiple degree-of-freedom positioning mechanism using wires (part 1). Int J Jpn Soc Precis Eng 28:131-138

Ming A, Higuchi T (1994) Study on multiple degree-of-freedom positioning mechanism using wires (part 2) -development of a planar completely restrained positioning mechanism-. Int J JSPE 28(3):235
Miskin MZ (2016) The automated design of materials far from equilibrium, 1 edn. Springer Theses. Springer International Publishing, Switzerland. https://doi.org/10.1007/978-3-319-24621-5

Miskin MZ, Jaeger HM (2013) Adapting granular materials through artificial evolution. Nat Mater 12:326-331. https://doi. org/10.1038/nmat3543

Murphy KA, Reiser N, Choksy D, Singer CE, Jaeger HM (2016) Freestanding loadbearing structures with z-shaped particles. Granul Matter 18(2):26. https://doi.org/10.1007/s10035-015-0600-2

Philipse AP (1996) The random contact equation and its implications for (colloidal) rods in packings, suspensions, and anisotropic powders. Langmuir 12(5):1127-1133. https://doi.org/10.1021/ la950671o

Piskorec L (2014) Remote material deposition. Space 562, envisaging the reality of digital fabrication: $3 \mathrm{D}$ printing and robotics pp 65

Tempel P, Pott A (2016) Parallele Seilroboter in Theorie und Praxis: Leichtbau. Energieeffizienz und Hohe Dynamiken als Potential, Elastizität als Hauptherausforderung

Tempel P, Schmidt A, Haasdonk B, Pott A (2018) Application of the rigid finite element method to the simulation of cable-driven parallel robots. In: Zeghloul S, Romdhane L, Laribi MA (eds) Computational kinematics: proceedings of the 7th international workshop on computational kinematics that was held at futuroscope-poitiers, France, in May 2017, mechanisms and machine science, vol. mechanisms and machine science. Springer International Publishing, Cham, pp 198-205. https://doi.org/10.1007/978-3319-60867-9_23

Trepanier M, Franklin SV (2010) Column collapse of granular rods. Phys Rev E 82(1):011308. https://doi.org/10.1103/PhysR evE.82.011308

Tsubaki K (2008) Tumbling units: tectonics of indeterminate extension. In: Borden GP, Meredith M (eds) Material matters: making architecture: the proceedings for the 2008 fall west ACSA conference. ACSA Press, Washington

Tsubaki K (2009) Tumbling units: tectonics of indeterminate extension. In: Crisman P, Gillem M (eds) The value of design: design is at the core of what we teach and practice: proceedings from the 2009 97th ACSA annual meeting. ACSA Press, Washington, pp 291-297

Tsubaki K (2011) Tumbling units: tectonics of indeterminate extension. In: Borden GP, Meredith M (eds) Matter: material processes in architectural production. Routledge, London, pp 187-203

Verhoeven R (2004) Analysis of the workspace of tendon-based stewart platforms. Doctoral dissertation, Universität Duisburg-Essen

Weisstein EW Circle-circle intersection. http://mathworld.wolfr am.com/Circle-CircleIntersection.html. Visited on 16/06/2019

Weisstein EW Sphere-sphere intersection. http://mathworld.wolfr am.com/Sphere-SphereIntersection.html. Visited on 16/06/2019

Wouterse A, Luding S, Philipse AP (2009) On contact numbers in random rod packings. Granul Matter 11(3):169-177. https://doi. org/10.1007/s10035-009-0126-6

Publisher's Note Springer Nature remains neutral with regard to jurisdictional claims in published maps and institutional affiliations. 